\title{
KECK SPECTROSCOPY OF $z>1$ FIELD SPHEROIDALS: DYNAMICAL CONSTRAINTS ON THE GROWTH RATE OF RED "NUGGETS"
}

\author{
Andrew B. Newman ${ }^{1}$, Richard S. Ellis ${ }^{1}$, Tommaso Treu 2,4 , and Kevin Bundy ${ }^{3,5}$ \\ ${ }^{1}$ Department of Astronomy, California Institute of Technology, Pasadena, CA 91125, USA; anewman@ astro.caltech.edu \\ 2 Department of Physics, University of California, Santa Barbara, CA 93106-9530, USA \\ ${ }^{3}$ Astronomy Department, University of California, Berkeley, CA 94705, USA \\ Received 2010 April 9; accepted 2010 June 4; published 2010 June 21
}

\begin{abstract}
We present deep Keck spectroscopy for 17 morphologically selected field spheroidals in the redshift range $1.05<z<1.60$ in order to investigate the continuity in physical properties between the claimed massive compact red galaxies ("nuggets") at $z \simeq 2$ and well-established data for massive spheroidal galaxies below $z \simeq 1$. By combining Keck-based stellar velocity dispersions with Hubble Space Telescope-based sizes, we find that the most massive systems $\left(M_{\text {dyn }}>10^{11} M_{\odot}\right)$ grew in size over $0<z<1.6$ as $(1+z)^{-0.75 \pm 0.10}($ i.e., $\times 2$ since $z=1.5)$ whereas intermediate mass systems $\left(10^{11} M_{\odot}>M_{\text {dyn }}>10^{10} M_{\odot}\right)$ did not grow significantly. These trends are consistent with a picture in which more massive spheroidals formed at higher redshift via "wetter" mergers involving greater dissipation. To examine growth under the favored "dry" merger hypothesis, we also examine size growth at a fixed velocity dispersion. This test, uniquely possible with our dynamical data, allows us to consider the effects of "progenitor bias." Above our completeness limit $\left(\sigma>200 \mathrm{~km} \mathrm{~s}^{-1}\right)$, we find size growth consistent with that inferred for the mass-selected sample, thus ruling out strong progenitor bias. To maintain continuity in the growth of massive galaxies over the past $10 \mathrm{Gyr}$, our new results imply that size evolution over $1.3<z<2.3$, a period of $1.9 \mathrm{Gyr}$, must have been even more dramatic than hitherto claimed if the red sources at $z>2$ are truly massive and compact.
\end{abstract}

Key words: galaxies: elliptical and lenticular, cD - galaxies: evolution

\section{INTRODUCTION}

The observation that many red galaxies with large stellar masses at $z \simeq 2$ are $3-5$ times more compact than equivalent ellipticals in the local universe (e.g., Daddi et al. 2005; Trujillo et al. 2007; van Dokkum et al. 2008; Buitrago et al. 2008; Damjanov et al. 2009) has been a source of much puzzlement. How can an early galaxy grow primarily in physical size without accreting significant stellar mass as required if these objects are the precursors of the most massive ellipticals observed today? Furthermore, studies of the fundamental plane and other stellar population indicators do not permit substantial recent star formation since $z \sim 2$ in massive galaxies, thus precluding growth by accretion of young stars or via gasrich ("wet") mergers (e.g., Treu et al. 2005, hereafter T05). Some have questioned the reliability of the observations, suggesting an underestimate of physical sizes or an overestimate of stellar masses (Hopkins et al. 2009; however, see Cassata et al. 2010 for a contrasting view). Others have proposed size expansion driven by self-similar dissipationless "dry" mergers, or mass accretion from minor mergers (Khochfar \& Silk 2006; Naab et al. 2009; Hopkins et al. 2010a, and references therein).

To verify the compact nature of distant sources and to track their evolution in size and mass, it is preferable to use dynamical masses $M_{\text {dyn }}$ from absorption line spectra, which do not suffer from uncertainties associated with the assumed initial mass function (IMF) and stellar mass estimates derived from broadband photometry (e.g., Muzzin et al. 2009). $M_{\text {dyn }}$ measurements are available for relatively large samples out to $z \sim 1$ (T05; van der Wel et al. 2008, hereafter vdW08), suggesting a small but detectable difference in average size

\footnotetext{
4 Packard Fellow.

5 Hubble Fellow.
}

at fixed mass when compared with the local universe. But beyond $z \simeq 1$, there is little high-quality dynamical data for field spheroidals. van Dokkum et al. (2009) undertook a heroic observation of a single $z>2$ source with a stellar mass $\simeq 2 \times 10^{11} M_{\odot}$ and an effective radius $r_{e}=0.8 \mathrm{kpc}$ typical of compact galaxies at $z \simeq 2.3$. The spectrum has a claimed stellar velocity dispersion of $\sigma=510_{-95}^{+165} \mathrm{~km} \mathrm{~s}^{-1}$, suggesting a remarkably dense system. van Dokkum et al. postulate the initial dissipative collapse at $z \simeq 3$ of a high mass "core" but are unable to account for its subsequent evolution onto the $z \simeq 1$ scaling relations. The quantitative effect of minor mergers on the physical size of a galaxy involves many variables, and it is unclear whether such dramatic size evolution is possible while maintaining the tightness of the fundamental plane and its projections (Nipoti et al. 2009).

Interpretation of the observed trends at fixed $M_{\mathrm{dyn}}$ is further complicated by the so-called progenitor bias (van der Wel et al. 2009): if galaxies grow by dry mergers, the main progenitor of a present-day massive galaxy did not have the same mass at $z \sim 2$. Similarly, if galaxies become recognizable as spheroidals only above a certain threshold in stellar velocity dispersion $\sigma_{\mathrm{ET}}$ that depends on redshift, it is clear that the addition of a new-and less dense-population could mimic a false evolutionary trend. This bias can be reduced by considering galaxy sizes at fixed $\sigma$. Foremost, $\sigma$ changes very little under a variety of growth mechanisms (e.g., Hopkins et al. 2010a) and it is therefore a better "label" than $M_{\text {dyn }}$ to track the assembly history. Second, $\sigma$ is closely correlated with stellar age (van der Wel et al. 2009) and therefore offers the most direct way to track the evolving population.

Given that there is no clear consensus in understanding the continuity between the galaxy population at $z<1$ and that at $z>2$, we have embarked on a campaign to measure $\sigma$ and $M_{\text {dyn }}$ for a large sample of field spheroidals at $1<z<1.7$. 
This has recently become practical using multi-object optical spectrographs equipped with deep depletion red-sensitive CCDs. Our goal is to extend the earlier work at $z<1$ (T05, vdW08) to within $\simeq 1$ Gyr of the sample of ultracompact galaxies at $z \simeq 2.3$. In this first analysis, we present new results spanning the redshift range $1.05<z<1.60$.

We adopt a $\Lambda$ CDM cosmology with $\left(\Omega_{m}, \Omega_{v}, h\right)=$ $(0.3,0.7,0.7)$; all magnitudes are in the AB system. A Chabrier IMF is assumed where necessary.

\section{SAMPLE AND OBSERVATIONS}

Our targets were selected from archival Hubble Space Telescope/Advanced Camera for Surveys (HST/ACS) data in the Extended Groth Strip (EGS; GO 10134, PI: Davis), SSA22 (GO 9760, PI: Abraham \& GO 10403, PI: Chapman), and GOODS-N (PI: Giavalisco) fields. For the EGS, we used the Bundy et al. (2006) catalog, which matches Canada-France-Hawaii Telescope (BRI, Coil et al. 2004; ugriz, CFHTLS) and Palomar $\left(J K_{s}\right)$ photometry. Photometric redshifts are supplemented by spectroscopic redshifts from the DEEP2 survey. For SSA22, we used a photometric redshift catalog based on Subaru $(B V R I z)$ and UH $2.2 \mathrm{~m}\left(J H K_{s}\right)$ imaging kindly provided by Capak (2004). In GOODS-N, we used the Bundy et al. (2009) catalog, which matches ACS and Subaru $K_{s}$ photometry. Galactic extinction corrections were based on the dust maps of Schlegel et al. (1998). The parent sample for spectroscopic study in EGS and SSA22 was defined by $I-K_{s}>2$, $I<23.5$, and $z>1$; in GOODS-N, the photometric criteria were F850LP $-K_{s}>1.5$ and F850LP $<23.5$. All galaxies satisfying these criteria were visually inspected in the ACS images by one of us (R.S.E.) and those with E/S0 or early-disk morphology retained.

Keck I LRIS observations were made for 14 EGS and SSA22 targets on 2009 June 26-28 in a median seeing of $0{ }^{\prime} 9$. The $600 \mathrm{~mm}^{-1}$ grating blazed at $1 \mu \mathrm{m}$ was used, providing a velocity resolution of $\sigma_{\text {inst }}=58 \mathrm{~km} \mathrm{~s}^{-1}$ at $9000 \AA$. The total integration times were $40.8 \mathrm{ks}$ and $32.4 \mathrm{ks}$ in the EGS and SSA22 fields, respectively. On 2010 April 5-6, LRIS observations were made of seven GOODS-N targets with $34.8 \mathrm{ks}$ of integration in $0{ }^{\prime} 8$ seeing. One additional GOODS-N spectrum was secured with Keck II DEIMOS observations on 2010 April 11-12 using the $831 \mathrm{~mm}^{-1}$ grating. The LRIS data were reduced using the code developed by Kelson (2003). Spectra were extracted using optimal weighting based on Gaussian fits to the spatial profile. Telluric absorption correction and relative flux calibration were provided by a DA star observed at matching airmass at the end of each night.

\section{VELOCITY DISPERSIONS AND PHOTOMETRY}

We measured stellar velocity dispersions, $\sigma$, by fitting broadened stellar templates using the PPXF code of Cappellari \& Emsellem (2004). The instrumental resolution was measured using unblended sky lines; their variation with wavelength was well fit by a low-order polynomial. The template collection comprised 348 stars of type F0-G9 from the Indo-US coudé library (Valdes et al. 2004) with a range of metallicities and luminosities (classes III-V). We verified that including A star templates does not affect our measurements. For each galaxy, PPXF constructed an optimal template as a linear combination of these stellar spectra, although our results do not significantly differ if the best-fitting single template is used. To avoid systematic errors, we masked pixels contaminated by $\mathrm{OH}$ emission. Based on tests with the continuum filtering, sky masking threshold, and stellar template choices, we assigned a systematic uncertainty to each velocity dispersion, typically $5 \%-10 \%$. We were able to secure a reliable dispersion for 17/22 galaxies (see Figure 1). Velocity dispersions were corrected to an effective circular aperture of radius $R_{e} / 8$ as described in Treu et al. (1999); the mean correction factor is 1.13 .

Surface photometry was measured in the HST images using GALFIT (Peng et al. 2002) with a point-spread function determined from a nearby isolated star. F814W imaging was used in EGS and SSA22, while F850LP data were adopted in GOODS-N. For consistency with the local Sloan Digital Sky Survey (SDSS) sample, we fit de Vaucouleurs profiles and determine circularized radii. We also fit Sérsic profiles but found that the mean Sérsic index $n$ is consistent with 4 (i.e., de Vaucouleurs). We estimate uncertainties of $\sim 10 \%$ in $R_{e}$ based on testing the background level, simulating the recovery of synthetic de Vaucouleur profiles placed in blank sky patches, and comparing with the independent measurements of vdW08 for the T05 subsample.

We convert the observed ACS magnitude to the rest $B$ magnitude by matching the observed $I-K_{s}$ color to a grid of Bruzual \& Charlot (2003) single-burst models of varying age and metallicity. The uncertainty in this $k$-correction is $\sim 4 \%$. Based on the optical and NIR photometry discussed in Section 2, stellar masses were estimated using the Bayesian stellar population analysis code developed by Auger et al. (2009). An exponentially decaying star formation history was assumed. Table 1 summarizes the dynamical and photometric properties for our sample of $17 z>1$ galaxies.

Our data allow us to compare photometrically derived stellar masses to dynamical masses, which we define as $M_{\mathrm{dyn}}=5 \sigma^{2} R_{e} / G$. Overall these are in good agreement: $\left\langle\log M_{\mathrm{dyn}} / M_{*}\right\rangle=0.17 \pm 0.07$, consistent with the difference between local dynamical and stellar masses inferred by Cappellari et al. (2006) and with the dark matter fractions (or heavier IMFs) found by independent methods (e.g., Graves \& Faber 2010; Treu et al. 2010).

\section{SIZE EVOLUTION}

Size evolution has commonly been studied by comparing spheroids of the same mass at different epochs. Although an unlikely evolutionary path for individual galaxies, it is the most observationally convenient approach, particularly at $z>1.5$, where dynamical measures are scarce. We first conduct this comparison in Section 4.1. Following our discussion in Section 1, we then examine size evolution at fixed velocity dispersion in Section 4.2.

\subsection{Size Evolution at Fixed Mass}

We constructed a local reference sample for comparison purposes using SDSS DR7 spectroscopic data (Stoughton et al. 2002). Red sequence galaxies with $0.05<z<0.15$ were selected using a color cut (Equation (1) of Yan et al. 2006). Velocity dispersions were corrected to a standard aperture of $R_{e} / 8$ as in Jorgensen et al. (1995). The resulting mean dynamical mass-radius relation is $R_{e}=2.88\left(M_{\mathrm{dyn}} / 10^{11} M_{\odot}\right)^{0.55} \mathrm{kpc}$, based on de Vaucouleurs radii measured in SDSS and interpolated to rest $B$ band. ${ }^{6}$

\footnotetext{
6 The relation is consistent with that of vdW08, taking into account the different apertures to which we normalize velocity dispersions.
} 

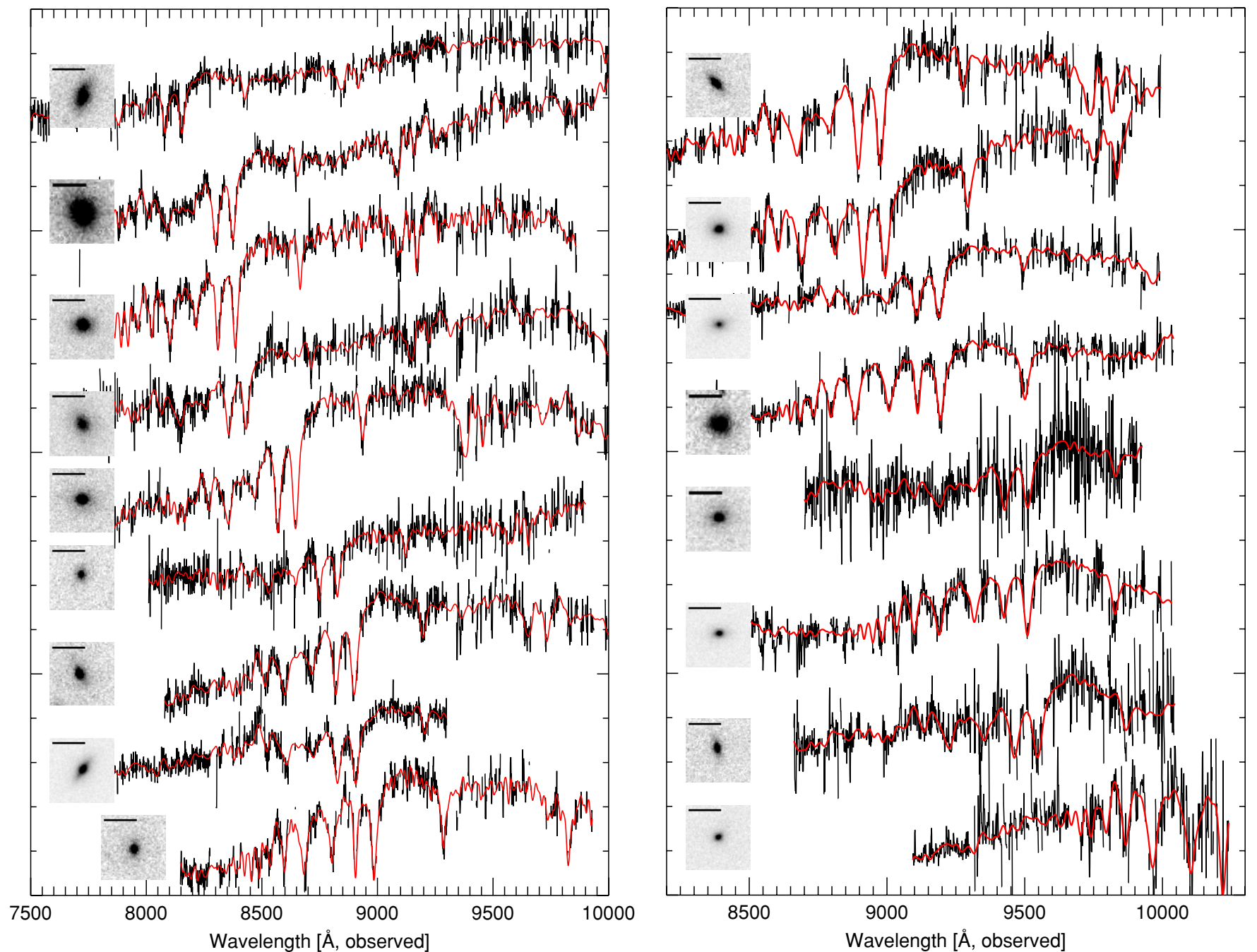

Figure 1. Keck spectra of our 17 targets ordered by redshift. Each is smoothed with a 3 pixel ( $2.4 \AA$ ) running median with sky lines omitted (black) and compared to fits to broadened stellar templates (red). HST images are inset with a $1^{\prime \prime}$ ruler. The order of objects matches that in Table 1.

Table 1

Photometric and Spectroscopic Data

\begin{tabular}{|c|c|c|c|c|c|c|c|c|c|c|c|c|}
\hline Name & R.A. & Decl. & Morph. & $z$ & $R_{e}$ & $\sigma$ & $M_{B}$ & $I-K_{s}$ & $z-K_{s}$ & $K_{S}$ & $\log M_{\text {dyn }} / M_{\odot}$ & $\log M_{*} / M_{\odot}$ \\
\hline E1 & 214.9853 & 52.9513 & S0? & 1.054 & 6.44 & $228 \pm 32$ & -22.32 & 2.14 & 1.70 & 19.79 & $11.59 \pm 0.13$ & 11.17 \\
\hline S1 & 334.3529 & 0.2734 & E & 1.110 & 4.74 & $242 \pm 18$ & -22.72 & 2.63 & 1.81 & 19.58 & $11.51 \pm 0.07$ & 11.23 \\
\hline E2 & 214.9702 & 52.9911 & E/S0 & 1.113 & 4.02 & $151 \pm 15$ & -22.18 & 2.15 & 1.69 & 20.16 & $11.03 \pm 0.09$ & 10.91 \\
\hline E3 & 215.0061 & 52.9755 & Sab & 1.124 & 6.11 & $266 \pm 28$ & -22.20 & 2.40 & 2.01 & 20.04 & $11.70 \pm 0.09$ & 11.01 \\
\hline E4 & 214.9847 & 52.9614 & E/S0 & 1.179 & 2.65 & $258 \pm 19$ & -22.21 & 2.23 & 1.76 & 20.02 & $11.31 \pm 0.07$ & 11.00 \\
\hline E5 & 214.9815 & 52.9501 & $\mathrm{E}$ & 1.225 & 1.43 & $139 \pm 25$ & -21.20 & 2.45 & 1.93 & 21.16 & $10.51 \pm 0.16$ & 10.57 \\
\hline E6 & 215.0351 & 52.9830 & So & 1.243 & 2.38 & $302 \pm 29$ & -22.20 & 2.57 & 2.06 & 19.79 & $11.40 \pm 0.08$ & 11.19 \\
\hline $\mathrm{GN} 1^{\mathrm{a}}$ & 189.2681 & 62.2264 & $\mathrm{Sab}$ & 1.253 & 1.29 & $290 \pm 26$ & -22.19 & 2.79 & 2.07 & 20.26 & $11.10 \pm 0.08$ & 11.07 \\
\hline $\mathrm{E} 7^{\mathrm{b}}$ & 215.1319 & 53.0163 & E/S0 & 1.262 & 1.57 & $103 \pm 21$ & -21.83 & 2.58 & 2.02 & 20.43 & $10.29 \pm 0.18$ & 10.90 \\
\hline E8 & 215.1371 & 53.0173 & Sab & 1.262 & 1.38 & $280 \pm 34$ & -21.90 & 2.38 & 1.90 & 20.76 & $11.10 \pm 0.11$ & 10.91 \\
\hline GN2 & 189.0634 & 62.1623 & $\mathrm{E} / \mathrm{S} 0$ & 1.266 & 1.58 & $239 \pm 36$ & -22.24 & 2.54 & 1.74 & 20.55 & $11.02 \pm 0.13$ & 10.88 \\
\hline GN3 & 188.9345 & 62.2068 & E/S0 & 1.315 & 3.44 & $288 \pm 28$ & -22.32 & 2.77 & 2.07 & 20.45 & $11.52 \pm 0.09$ & 11.01 \\
\hline $\mathrm{S} 2^{\mathrm{b}}$ & 334.3502 & 0.3032 & $\mathrm{Sab}$ & 1.315 & 2.47 & $171 \pm 29$ & -22.37 & 2.29 & 1.51 & 20.80 & $10.93 \pm 0.15$ & 10.69 \\
\hline S3 & 334.4233 & 0.2256 & $\mathrm{E} / \mathrm{S} 0$ & 1.394 & 2.50 & $271 \pm 71$ & -22.01 & 3.20 & 2.44 & 20.74 & $11.33 \pm 0.23$ & 11.09 \\
\hline $\mathrm{GN} 4^{\mathrm{b}}$ & 189.1132 & 62.1325 & $\mathrm{E}$ & 1.395 & 0.77 & $206 \pm 49$ & -21.93 & 2.24 & 1.71 & 21.24 & $10.58 \pm 0.21$ & 10.55 \\
\hline E9 & 215.1219 & 52.9576 & S0/a & 1.406 & 1.19 & $337 \pm 79$ & -22.02 & 2.39 & 2.19 & 20.82 & $11.20 \pm 0.21$ & 10.83 \\
\hline $\mathrm{GN}^{\mathrm{b}}$ & 188.9625 & 62.2286 & $\mathrm{E}$ & 1.598 & 0.68 & $274 \pm 41$ & -22.51 & 2.71 & 2.25 & 20.69 & $10.77 \pm 0.13$ & 11.02 \\
\hline
\end{tabular}

Notes. R.A. and decl. are in the $\mathrm{J} 2000$ equinox. $R_{e}$ is in kpc. Velocity dispersions (in $\mathrm{km} \mathrm{s}^{-1}$ ) are standardized to a circular aperture of radius $R_{e} / 8$, and uncertainties include the systematic component added in quadrature. $M_{B}$ is the rest-frame $B$-band absolute magnitude. The $I-K_{s}$ and $z-K_{s}$ photometry has been interpolated to a uniform system of Bessell $I$ and SDSS $z$. Dynamical mass uncertainties include the total uncertainty (statistical and systematic) in $\sigma$. Stellar masses assume a Chabrier IMF. The order of galaxies (by redshift) matches Figure 1.

a The DEIMOS observation.

${ }^{\mathrm{b}}$ Systems with strong Balmer absorption. 


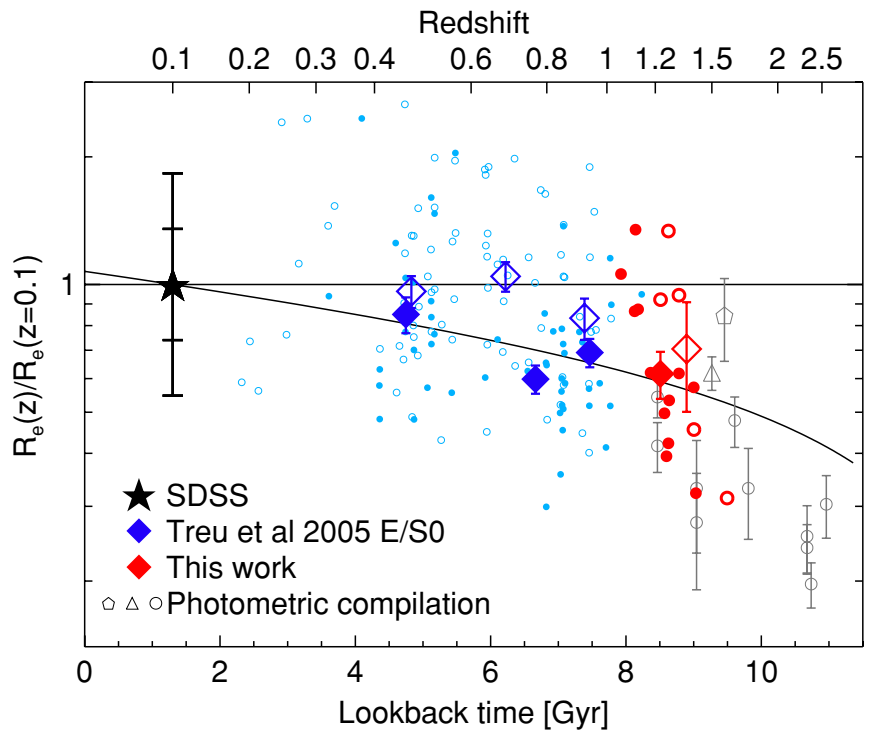

Figure 2. Size evolution of spheroids at fixed mass. Solid diamonds show the mean size and its uncertainty for massive $\left(\log M_{\mathrm{dyn}} / M_{\odot}>11\right)$ spheroids relative to red sequence galaxies in SDSS of the same dynamical mass (computed in log space). Open diamonds refer to intermediate-mass $(10<$ $\left.\log M_{\text {dyn }} / M_{\odot}<11\right)$ systems. The solid line shows a $(1+z)^{-0.75 \pm 0.10}$ fit to the more massive sample. Individual galaxies in the spectroscopic samples are shown by colored circles. Light gray points with error bars are based on photometric stellar masses and show mean sizes relative to SDSS galaxies of the same stellar mass, based on the Shen et al. (2003) relation, for several samples: Saracco et al. (2010, triangle), Mancini et al. (2010, pentagon), and the vdW08 (circles) compilation.

Figure 2 shows the size evolution inferred by comparing the present sample and the T05 E/S0 galaxies to this SDSS relation. The T05 and present samples are well-matched in morphology and rest optical colors and so comprise an excellent data set for studying evolution over a wide redshift interval. A simple power law fit $(1+z)^{-0.75 \pm 0.10}$ to the $M_{\text {dyn }}>10^{11} M_{\odot}$ sample is indicated by the solid line. This corresponds to a $40 \%$ decrease in size by $z=1$, and marginally slower evolution than that inferred by vdW08 for $M_{\mathrm{dyn}}>3 \times 10^{10} M_{\odot}$ spheroidals $\left((1+z)^{-0.98 \pm 0.11}\right.$; corresponding to a $50 \%$ decrease at $\left.z=1\right)$. However, the difference is partly explained by the correction applied by vdW08 to their measured sizes based on simulations. In contrast, we do not apply any corrections to our measured sizes given the lack of consensus on this matter in the literature (see also Hopkins et al. 2009; Mancini et al. 2010) and the results of our own simulations.

Our $\langle z\rangle=1.3$ sample probes an epoch within $2 \mathrm{Gyr}$ of the $z \sim 2.3$ samples whose compact sizes have motivated the present work. Figure 2 illustrates that, given the size dispersion in the dynamical sample at a given redshift and the difficulties of comparing our dynamical sample with one whose masses are likely less precise, the sharp drop in size seen over this short time interval may not be that significant. If confirmed, however, the implied size evolution is quite large compared to the expected accretion from mergers over the same period, which we estimate to be $\sim 40 \%$ of a typical $10^{11} M_{\odot}$ galaxy at $z=1.3{ }^{7}$ Thus, if minor mergers are responsible, these results imply a very high efficiency of $d \log R_{e} / d \log M \simeq 2.6$ for growing spheroids, just consistent with the upper end of estimates determined from merger simulations (e.g., Hopkins et al. 2010a).

\footnotetext{
Estimated using the merger rate calculator presented in Hopkins et al (2010b).
}

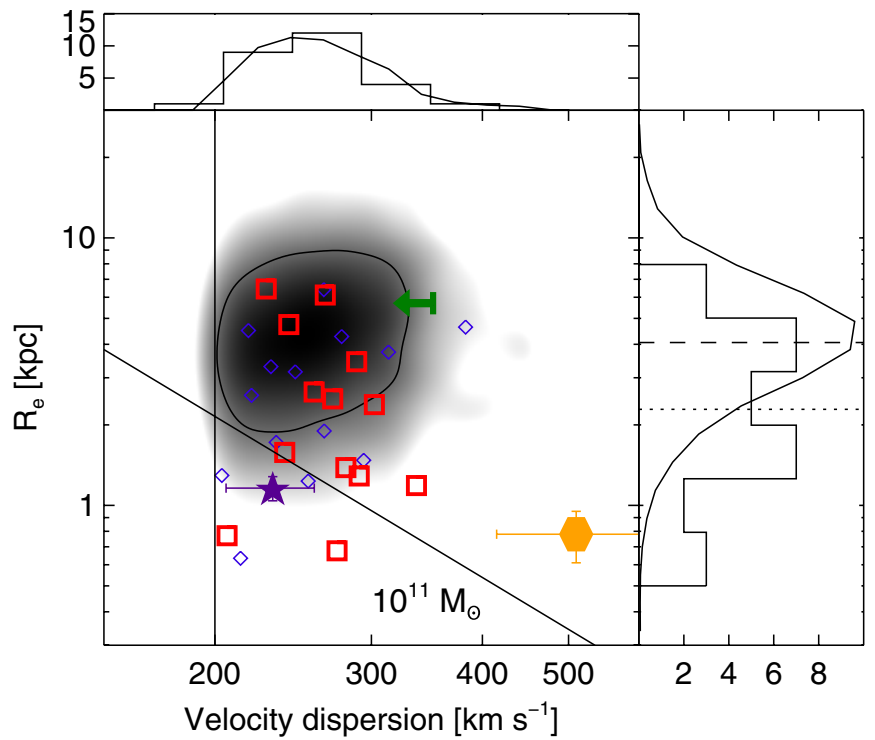

Figure 3. Distribution of effective radii at matched velocity dispersion for spheroids with $\sigma>200 \mathrm{~km} \mathrm{~s}^{-1}$ in the present sample (red), the T05 $z>0.9$ sample (blue), and SDSS (smoothed gray scale with $1 \sigma$ contour). The histogram (this data) and the solid line (SDSS) in the top panel agree by construction. The right panel compares the distribution of $R_{e}$ for the matched samples. Dashed and dotted lines indicate the means in log space. For comparison, the $z \sim 1.7$ stacked sample of Cappellari et al. (2009) is shown (star), along with the single galaxies of van Dokkum et al. (2009, hexagon, $z=2.186)$ and Onodera et al. (2010, green upper limit, $z=1.823$ ). The diagonal line represents $M_{\mathrm{dyn}}=$ $10^{11} M_{\odot}$.

Also striking is the different trend seen in less massive $10<\log M_{\mathrm{dyn}} / M_{\odot}<11$ galaxies (open diamonds in Figure 2). Although the high- $z$ samples are small and include some compact examples, we find no evidence for mean size evolution over $0<z<1.6$, i.e., $\propto(1+z)^{0.02 \pm 0.15}$. This is consistent with a picture in which more massive galaxies formed earlier and from wetter mergers with more dissipation, creating more compact remnant spheroids (e.g., Khochfar \& Silk 2006; Trujillo et al. 2006), and at variance with the model proposed by van der Wel et al. (2009) in which lower-mass galaxies are most strongly affected by progenitor bias and display the strongest evolution. However, we caution that the lower mass samples may be affected by selection effects, since the brighter-and therefore possibly larger - objects may be preferentially selected given our flux limits. This is not a concern for the $>10^{11} M_{\odot}$ sample, where we are complete for any reasonable mass-to-light ratio. A characterization of the bias requires a self-consistent model with Monte Carlo simulations, which is beyond the scope of this Letter and is left for future work when larger samples will be available.

\subsection{Size Evolution at Fixed Velocity Dispersion}

Comparisons at fixed mass may be affected by "progenitor bias." A preferred approach, when dynamical data are available, is to examine galaxies of the same velocity dispersion. This offers the two advantages discussed in Section 1 and is illustrated in Figure 3. A cut in $M_{\mathrm{dyn}}$ includes only the largest galaxies at a fixed $\sigma$. Therefore, if galaxies below some threshold $\sigma_{\mathrm{ET}}$ are missing from the high-redshift samples, this could mimic an evolutionary trend in mass-selected samples. According to the preferred prescription of van der Wel et al. (2009), $\sigma_{\mathrm{ET}}=233 \mathrm{~km} \mathrm{~s}^{-1}$ at $z=1.3$. Therefore we should expect to see some effect for our sample, even though the presence of lower $\sigma$ 
objects in our sample already suggests that the progenitor bias is not as strong.

Figure 3 shows this comparison in terms of the $\sigma-R_{e}$ plane. To avoid luminosity selection biases, we consider only galaxies with $\sigma>200 \mathrm{~km} \mathrm{~s}^{-1}$, where we are $90 \%$ complete, based on the SDSS distribution of luminosity at fixed $\sigma$ and a conservative estimate of luminosity evolution, consistent with passive evolution of an old stellar population formed at $z_{f}=3$. The SDSS galaxies (gray scale) are weighted so as to match the $\sigma$ distribution of the $0.9<z<1.6$ sample (top panel). The right panel of Figure 3 then compares the size distributions of the local and $0.9<z<1.6$ samples at matching $\sigma$. By fitting for size evolution at fixed $\sigma$ we find that sizes evolve as $(1+z)^{-0.88 \pm 0.19}$. The good agreement with the size evolution inferred at fixed mass rules out strong progenitor bias. A full evolutionary model with selection effects is needed to include objects with lower $\sigma$ and quantify progenitor bias and size evolution more accurately. This is left for future work with larger samples.

\section{CONCLUSIONS}

Our Keck spectra have shown the utility of securing individual spectroscopic and photometric measures for a representative sample of $z>1$ massive spheroidals. By probing to $z \simeq 1.6$, we are sampling velocity dispersions, sizes, and dynamical masses within 1.2 Gyr of the puzzling population of compact red galaxies at $z \simeq 2-2.3$.

Importantly, the size evolution we infer over $0<z<1.6$ at fixed dynamical mass is modest: $\simeq \times 2$ for the most massive $\left(\log M_{\text {dyn }} / M_{\odot}>11\right)$ examples but much smaller for lower mass systems. If the compact red galaxies at $z \simeq 2-2.3$ are their precursors, they must have grown dramatically in size over a very short time interval.

We thank Peter Capak for kindly providing photometric catalogs for the SSA22 field and Arjen van der Wel for helpful comments regarding size measurements. It is a pleasure to acknowledge Matt Auger for computing the stellar masses and Dan Stark for the DEIMOS observation. We thank the anonymous referee for comments that improved the quality of this Letter. We are grateful to the staff of the Keck Observatory and Connie Rockosi, in particular, for ensuring our early access to the upgraded LRIS-R was successful. Research support by the Packard Foundation is gratefully acknowledged by T.T. The authors recognize and acknowledge the cultural role and reverence that the summit of Mauna Kea has always had within the indigenous Hawaiian community. We are most fortunate to have the opportunity to conduct observations from this mountain.

\section{REFERENCES}

Auger, M. W., Treu, T., Bolton, A. S., Gavazzi, R., Koopmans, L. V. E., Marshall, P. J., Bundy, K., \& Moustakas, L. A. 2009, ApJ, 705, 1099

Bruzual, G., \& Charlot, S. 2003, MNRAS, 344, 1000

Buitrago, F., Trujillo, I., Conselice, C. J., Bouwens, R. J., Dickinson, M., \& Yan, H. 2008, ApJ, 687, L61

Bundy, K., Fukugita, M., Ellis, R. S., Targett, T. A., Belli, S., \& Kodama, T. 2009, ApJ, 697, 1369

Bundy, K., et al. 2006, ApJ, 651, 120

Capak, P. L. 2004, PhD thesis, University of Hawaii

Cappellari, M., \& Emsellem, E. 2004, PASP, 116, 138

Cappellari, M., et al. 2006, MNRAS, 366, 1126

Cappellari, M., et al. 2009, ApJ, 704, L34

Cassata, P., et al. 2010, ApJ, 714, L79

Coil, A. L., Newman, J. A., Kaiser, N., Davis, M., Ma, C., Kocevski, D. D., \& Koo, D. C. 2004, ApJ, 617, 765

Daddi, E., et al. 2005, ApJ, 626, 680

Damjanov, I., et al. 2009, ApJ, 695, 101

Graves, G. J., \& Faber, S. M. 2010, arXiv:1005.0014

Hopkins, P. F., Bundy, K., Hernquist, L., Wuyts, S., \& Cox, T. J. 2010a, MNRAS, 401, 1099

Hopkins, P. F., Bundy, K., Murray, N., Quataert, E., Lauer, T. R., \& Ma, C. 2009, MNRAS, 398, 898

Hopkins, P. F., et al. 2010b, ApJ, 715, 202

Jorgensen, I., Franx, M., \& Kjaergaard, P. 1995, MNRAS, 276, 1341

Kelson, D. D. 2003, PASP, 115, 688

Khochfar, S., \& Silk, J. 2006, ApJ, 648, L21

Mancini, C., et al. 2010, MNRAS, 401, 933

Muzzin, A., Marchesini, D., van Dokkum, P. G., Labbé, I., Kriek, M., \& Franx, M. 2009, ApJ, 701, 1839

Naab, T., Johansson, P. H., \& Ostriker, J. P. 2009, ApJ, 699, L178

Nipoti, C., Treu, T., Auger, M. W., \& Bolton, A. S. 2009, ApJ, 706, L86

Onodera, M., et al. 2010, ApJ, 715, L6

Peng, C. Y., Ho, L. C., Impey, C. D., \& Rix, H. 2002, AJ, 124, 266

Saracco, P., Longhetti, M., \& Gargiulo, A. 2010, arXiv:1004.3403

Schlegel, D. J., Finkbeiner, D. P., \& Davis, M. 1998, ApJ, 500, 525

Shen, S., Mo, H. J., White, S. D. M., Blanton, M. R., Kauffmann, G., Voges, W., Brinkmann, J., \& Csabai, I. 2003, MNRAS, 343, 978

Stoughton, C., et al. 2002, AJ, 123, 485

Treu, T., Auger, M. W., Koopmans, L. V. E., Gavazzi, R., Marshall, P. J., \& Bolton, A. S. 2010, ApJ, 709, 1195

Treu, T., Stiavelli, M., Casertano, S., Møller, P., \& Bertin, G. 1999, MNRAS, 308, 1037

Treu, T., et al. 2005, ApJ, 633, 174

Trujillo, I., Conselice, C. J., Bundy, K., Cooper, M. C., Eisenhardt, P., \& Ellis, R. S. 2007, MNRAS, 382, 109

Trujillo, I., et al. 2006, ApJ, 650, 18

Valdes, F., Gupta, R., Rose, J. A., Singh, H. P., \& Bell, D. J. 2004, ApJS, 152, 251

van der Wel, A., Bell, E. F., van den Bosch, F. C., Gallazzi, A., \& Rix, H. 2009, ApJ, 698, 1232

van der Wel, A., Holden, B. P., Zirm, A. W., Franx, M., Rettura, A., Illingworth, G. D., \& Ford, H. C. 2008, ApJ, 688, 48

van Dokkum, P. G., Kriek, M., \& Franx, M. 2009, Nature, 460, 717

van Dokkum, P. G., et al. 2008, ApJ, 677, L5

Yan, R., Newman, J. A., Faber, S. M., Konidaris, N., Koo, D., \& Davis, M. 2006, ApJ, 648, 281 